\title{
Qualidade de vida de crianças com deficiência visual atendidas em Ambulatório de Estimulação Visual Precoce
}

\author{
Quality of life in visual impaired children treated for Early Visual Stimulation
}

Alcione Aparecida Messa ${ }^{1}$, Célia Regina Nakanami², Marcia Caires Bestilleiro Lopes ${ }^{3}$

\section{RESUMO}

Objetivo: Analisar a qualidade de vida em crianças com deficiência visual atendidas em Ambulatório de Estimulação Visual Precoce da Unifesp em duas situações, antes e após intervenções reabilitacionais pela equipe multiprofissional.

Métodos: Aplicou-se o questionário de qualidade de vida QFVI. O instrumento possui uma versão para crianças menores de três anos e outra para crianças maiores de três anos (três a sete anos) e é dividido em seis domínios: Saúde geral; Saúde geral da visão; Competência; Personalidade; Impacto familiar e Tratamento,

Resultados: A correlação entre as subescalas nos dois momentos mostrou-se significativa. Houve diferença estatisticamente significativa em saúde geral da visão $(p=0,029)$ e outras diferenças importantes foram obtidas em saúde geral, impacto familiar e qualidade de vida geral.

Discussão: Este instrumento mostrou-se eficiente na aferição da qualidade de vida relacionada à visão nas famílias que frequentam esse ambulatório. As intervenções multidisciplinares promoveram melhora da visão funcional da criança e qualidade de vida da família como um todo.

Conclusão: A qualidade de vida relacionada à visão de crianças atendidas no Ambulatório de Estimulação Visual Precoce da Unifesp apresentou melhora significativa na saúde geral da visão.

Descritores: Qualidade de vida; Baixa visão/reabilitação; Serviços de reabilitação

\section{ABSTRACT}

Purpose: To evaluate the quality of life in visually impaired children followed in the Early Visual Stimulation Ambulatory of Unifesp in two moments, before and after rehabilitational intervention of multiprofessional team.

Methods: A CVFQ quality of life questionnaire was used. This instrument has a version for less than threeyears old children and another one for children older than threeyears (three to seven years) divided in six subscales: General health, General vision health, Competence, Personality, Family impact and Treatment.

Results: The correlation between the subscales on two moments was significant. There was a statistically significant difference in general vision health $(p=0,029)$ and other important differences obtained in general health, family impact and quality of life general score.

Discussion: The questionnaire showed to be effective in order to measure the quality of life related to vision on families followed on this ambulatory. The multidisciplinary interventions provided visual function and familiar quality of life improvement.

Conclusion: The quality of life related to vision in children followed in Early Visual Stimulation Ambulatory of Unifesp showed a significant improvement on general vision health.

Keywords: Quality of life; Vision, low/rehabilitation; Rehabilitation services

\section{INTRODUÇÃO}

A percepção visual é uma das principais vias de veiculação e acesso às informações que são utilizadas posteriormente na construção de representações mentais e um dos mais importantes sentidos no desenvolvimento neuropsicomotor da criança. A deficiência na visão diminui a capacidade de integração de tais informações, afetando a personalidade do indivíduo, conduzindo-o a um ambiente social diferente do vidente ${ }^{(1,2)}$

As crianças com deficiência visual podem apresentar limitações no desempenho funcional de suas atividades de rotina diária, relacionadas ao autocuidado e mobilidade. Além disso, a deficiência visual pode interferir nos aspectos estruturais do indivíduo, constituição psíquica, personalidade, afetando de diferentes formas e em diferentes graus todas as suas funções, originando um processo de organização mental peculiar(3)

Em relação ao impacto familiar, as mães de crianças com deficiência visual relatam o profundo impacto emocional que a situação pode gerar. Sentem desespero, revolta, tristeza e raiva quando descobrem as dificuldades visuais de seus filhos, apresentando medo que a criança fique cega e pouco entendimento do diagnóstico e da baixa visão(4).

Não se deve afirmar, porém, que as famílias de crianças com deficiência necessariamente apresentarão distúrbios nos relacionamentos, dificuldades permanentes ou disfunções individuais. O risco se determina com a influência de múltiplos fatores, entre eles a capacidade da família em implementar estratégias e recursos de adaptação. O risco de distúrbios coexiste com a possibilidade de crescimento, fortalecimento e união dos membros familiares ${ }^{(5)}$.

Com o intuito de avaliar o impacto de tratamentos de saúde e percepções do paciente, os instrumentos de qualidade de vida têm obtido reconhecimento como uma importante ferramenta ${ }^{(6,7)}$. Em estudos referentes à qualidade de vida de crianças com deficiência visual, os resultados apontam comprometimento da qualidade de vida ${ }^{(8-10)}$, podendo também ocorrer comprometimento emocional
Submetido para publicação: 6 de março de 2012

Aceito para publicação: 18 de maio de 2012

Trabalho realizado no Departamento de Oftalmologia da Universidade Federal de São Paulo - Unifesp - Setor de Baixa Visão e Reabilitação Visual - Ambulatório de Estimulação Visual Precoce.

Psicóloga, Ambulatório de Estimulação Visual Precoce. Universidade Federal de São Paulo - Unifesp - São Paulo (SP), Brasil.

Médica, Setor de Baixa Visão e Reabilitação Visual. Departamento de Oftalmologia. Universidade

Federal de São Paulo - Unifesp - São Paulo (SP), Brasil.

Fisioterapeuta. Ambulatório de Estimulação Visual Precoce. Departamento de Oftalmologia.

Universidade Federal de São Paulo - Unifesp - São Paulo (SP), Brasil.
Financiamento: Não houve financiamento para este trabalho.

Divulgação de potenciais Conflitos de Interesse: A.A.Messa, Nenhum; C.R.Nakanami, Nenhum; M.C.B.Lopes, Empregada pela Universidade Federal de São Paulo - Unifesp.

Endereço para correspondência: Alcione Aparecida Messa. Rua Doutor Bacelar, 231, conjunto 31 - São Paulo - SP - 04026-000 - Brasil -E-mail: alcioneam@hotmail.com

Projeto aprovado pelo Comitê de Ética da Universidade Federal de São Paulo/Unifesp sob o no 1032/09. 
e dependência para a realização das tarefas cotidianas(11). Indivíduos com doenças oculares podem se mostrar mais retraídos, pessimistas, intuitivos e inseguros ${ }^{(12)}$.

Com base na literatura revisada, observa-se a importância de avaliar a eficácia de tratamentos para doenças oftalmológicas dos primeiros anos, tais como métodos de estimulação visual e reabilitação. As condutas reabilitacionais em crianças de baixa visão são importantes por promover a funcionalidade de resíduos de visão e a inclusão socioescolar ${ }^{(13)}$. O processo de reabilitação envolve condutas interdisciplinares com foco na redução das perdas funcionais, promoção da saúde, valorizando a independência e autonomia do paciente ${ }^{(8)}$.

O objetivo deste estudo foi avaliar a qualidade de vida relacionada à visão de crianças com deficiência visual atendidas em Ambulatório de Estimulação Visual Precoce em duas situações, antes e após intervenções reabilitacionais de equipe multiprofissional.

\section{MÉTODOS}

Este estudo de coorte foi realizado no período de abril/2009 a março/2010, no setor de Baixa Visão e Reabilitação Visual, Departamento de Oftalmologia da Unifesp - Ambulatório de Estimulação Visual Precoce. O estudo seguiu os princípios da Declaração de Helsinque, foi analisado e aprovado pelo Comitê de Ética em Pesquisa da Universidade Federal de São Paulo/Hospital São Paulo, sob o número 1032/09. Todos os participantes foram informados e orientados quanto aos objetivos e importância da pesquisa, leram o termo de consentimento livre e esclarecido (ou foi lido para os mesmos), concordando e assinando, permitindo assim a utilização dos dados para este estudo.

Foi utilizado o Questionário de Função Visual Infantil (QFVI), um instrumento validado para acessar o impacto da deficiência visual em crianças até sete anos e suas famílias. O instrumento possui uma versão para crianças menores de três anos (zero a dois anos e 11 meses) com 35 questões e outra para crianças maiores de três anos (três a sete anos) com 40 questões, abordando diversos aspectos sobre a qualidade da função visual infantil e é dividido em seis domínios: Saúde geral; Saúde geral da visão; Competência; Personalidade; Impacto familiar e Tratamento(14).

A administração do questionário foi feita em entrevista individual, com duração média de 15 a 20 minutos para o preenchimento completo, sendo o questionário aplicado antes e após intervenções de reabilitação (com um intervalo médio de três meses entre as duas aplicações). A reabilitação no Ambulatório de Estimulação Visual Precoce referido consiste em intervenções oftalmológicas, fisioterapêuticas, terapêuticas ocupacionais e psicológicas, centradas na necessidade da criança e da família, em atendimentos que incluíram a avaliação da visão funcional, conduta de estimulação visual precoce, suporte emocional familiar e controle médico.

Um participante foi excluído da pesquisa, por não continuidade de tratamento no ambulatório, impossibilitando a aplicação do questionário no segundo momento, após três meses de intervenção. Participaram deste estudo um total de 24 pais/cuidadores de crianças com deficiência visual que realizaram seguimento no Ambulatório de Estimulação Visual Precoce, distribuídos igualmente em dois grupos: crianças menores de três anos e crianças maiores de três anos.

A idade das crianças variou entre seis meses e seis anos (média = $34,6$ meses, $D P=20,65)$, sendo a maioria do sexo feminino $(66,7 \%)$ (Tabela 1). A maioria dos respondentes ao questionário foram mães (91,7\%), com idade na faixa de 21 a 30 anos (58,4\%), e escolaridade de ensino médio completo (54,2\%). Duas participantes eram avós da criança, duas mães tinham idade abaixo de 21 anos, seis mães tinham entre 31 e 40 anos e duas tinham acima de 50 anos. Quanto à escolaridade, três participantes tinham ensino fundamental incompleto, uma tinha ensino fundamental completo, quatro tinham ensino médio incompleto, uma tinha superior incompleto e duas tinham superior completo (Tabela 2).
As crianças obtiveram o diagnóstico de baixa visão por avaliação oftalmológica e realização dos exames de Teller e Potencial Visual Evocado (PVE) atestando visão reduzida, comprovado por CID (Classificação Internacional de doenças). Dentre os diagnósticos oftalmológicos, as crianças apresentaram: alterações de retina e nervo óptico (9), catarata (9), glaucoma (1) e alterações visuais corticais (5). Cabe ressaltar que dez crianças apresentavam alterações neurológicas e/ou síndromes associadas.

No Ambulatório de Estimulação Visual Precoce, as crianças foram submetidas à avaliação multidisciplinar para definir a conduta de tratamento e posterior inclusão na rotina do ambulatório, cuja frequência de atendimentos, semanal ou quinzenal, foi determinada de acordo com a necessidade de cada paciente.

Para a comparação dos questionários nos dois momentos, utilizou-se teste estatístico T pareado simples.

\section{RESULTADOS}

De acordo com a tabulação dos escores do questionário calculados em porcentagem (Tabela 3), obteve-se melhora na saúde geral da visão, competência e impacto familiar para o grupo de crianças menores de três anos. No grupo de crianças maiores de três anos, as mudanças foram obtidas em saúde geral da visão, impacto familiar e tratamento. O escore geral de qualidade de vida entre os dois momentos teve melhora em ambos os grupos.

De acordo com a análise estatística dos dados, a correlação entre as subescalas nos dois momentos do grupo de crianças menores de três anos foi significativa em saúde geral da visão $(p<0)$, competência $(p=0,001)$ e qualidade de vida geral $(p=0,001)$. No grupo de crianças maiores de três anos, a correlação entre as subescalas

\section{Tabela 1. Descrição das crianças por sexo, idade média e desvio} padrão

\begin{tabular}{llcc}
\hline $\begin{array}{l}\text { Características - } \\
\text { crianças }\end{array}$ & $\begin{array}{c}\text { Menores de } \\
\text { três anos }\end{array}$ & $\begin{array}{c}\text { Maiores de } \\
\text { três anos }\end{array}$ \\
\hline Sexo & Feminino & 10 & 6 \\
& Masculino & 2 & 6 \\
Idade $\quad$ Média & \multicolumn{2}{c}{34,6 meses } \\
& Desvio padrão & \multicolumn{2}{c}{20,65} \\
\hline
\end{tabular}

Tabela 2. Descrição das características das respondentes ao questionário por parentesco, idade e escolaridade

\begin{tabular}{|c|c|c|c|}
\hline \multicolumn{2}{|c|}{$\begin{array}{l}\text { Características - } \\
\text { respondentes ao questionário }\end{array}$} & \multirow{2}{*}{$\begin{array}{c}\begin{array}{c}\text { Menores de } \\
\text { três anos }\end{array} \\
11\end{array}$} & \multirow{2}{*}{$\begin{array}{c}\begin{array}{c}\text { Maiores de } \\
\text { três anos }\end{array} \\
11\end{array}$} \\
\hline Parentesco & Mãe & & \\
\hline & Avó & 1 & 1 \\
\hline \multirow[t]{4}{*}{ Idade } & Abaixo de 21 anos & 1 & 1 \\
\hline & 21-30 anos & 10 & 4 \\
\hline & $31-40$ anos & - & 6 \\
\hline & Acima de 50 anos & 1 & 1 \\
\hline \multirow[t]{6}{*}{ Escolaridade } & Ensino fundamental incompleto & & 3 \\
\hline & Ensino fundamental completo & & 1 \\
\hline & Ensino médio incompleto & & 4 \\
\hline & Ensino médio completo & & 3 \\
\hline & Ensino superior incompleto & & 1 \\
\hline & Ensino superior completo & & 2 \\
\hline
\end{tabular}


Tabela 3. Escores do questionário em porcentagem para os dois momentos

\begin{tabular}{|c|c|c|c|c|}
\hline \multirow{2}{*}{ Subescalas do QFVI } & \multicolumn{2}{|c|}{ Menores de três anos } & \multicolumn{2}{|c|}{ Maiores de três anos } \\
\hline & 1ํmomento & 2ㅇ momento & 1ำ momento & 2으omento \\
\hline Saúde geral & 70,8 & 68,8 & 62,5 & 52,1 \\
\hline Saúde geral da visão & 63,3 & 68,3 & 61,7 & 70,0 \\
\hline Competência & 64,0 & 70,5 & 68,4 & 65,1 \\
\hline Personalidade & 75,2 & 74,4 & 74,5 & 73,1 \\
\hline Impacto familiar & 41,7 & 47,2 & 49,0 & 53,1 \\
\hline Tratamento & 68,6 & 65,0 & 65,6 & 67,5 \\
\hline Qualidade de vida - escore total & 61,3 & 64,7 & 64,0 & 65,5 \\
\hline
\end{tabular}

foi significativa para as subescalas de saúde geral $(p=0,034)$, saúde geral da visão $(p=0,003)$, personalidade $(p=0,007)$ e qualidade de vida geral $(p<0)$.

Considerando-se a amostra total, ou seja, o grupo de maiores e menores de três anos (24 participantes), a correlação entre as subescalas nos dois momentos mostrou-se significativa em saúde geral $(p=0,008)$, saúde geral da visão $(p<0)$, competência $(p=0,006)$, personalidade $(p=0,031)$, impacto familiar $(p=0,030)$ e qualidade de vida geral $(p<0)$.

Em relação aos dois momentos, o grupo de crianças menores de três anos apresentou as diferenças mais importantes em saúde geral da visão $(p=0,111)$ e impacto familiar $(p=0,097)$. No grupo de crianças maiores de três anos as diferenças foram em saúde geral $(p=0,096)$ e saúde geral da visão $(p=0,127)$.

$\mathrm{Na}$ amostra total, entre os dois momentos, houve diferença estatisticamente significativa em saúde geral da visão $(p=0,029)$. Outras diferenças importantes foram obtidas em saúde geral $(p=0,162)$, impacto familiar $(p=0,101)$ e qualidade de vida geral $(p=0,091)$.

\section{DISCUSSÃO}

Os resultados obtidos de correlação entre as subescalas nos dois momentos antes e após intervenções reabilitacionais de equipe multiprofissional, com intervalos de três meses, indicam que o questionário é adequado para os fins da pesquisa. O instrumento mostrou-se eficiente nas medidas de qualidade de vida relacionada à visão das crianças com deficiência visual que frequentam o Ambulatório de Estimulação Visual Precoce.

Crianças com deficiência visual apresentam atraso global no desenvolvimento neuropsicomotor e na visão funcional, principalmente no comportamento da coordenação(15). A estimulação visual precoce é uma intervenção utilizada com essas crianças ${ }^{(13,16)}$, sendo considerada uma ação efetiva para propiciar resultados visuais mais consistentes no tratamento de doenças oculares ${ }^{(2,17)}$. Tem o objetivo de despertar o desejo e o interesse da criança para agir sobre o ambiente, funcionando também com caráter preventivo de deficiências secundárias. Quanto mais precoce o diagnóstico da deficiência visual e o início da estimulação, melhores as repercussões na eficiência visual e em todos os aspectos do desenvolvimento(18).

As mudanças clínicas de funcionalidade visual são observáveis com o decorrer do tratamento, conforme comprovadas pelo resultado obtido na presente investigação, em que houve melhora significativa da saúde geral da visão entre os dois momentos. As intervenções multidisciplinares promovem melhora da função visual da criança e da qualidade de vida da família como um todo.

Diante da pequena amostra do estudo (24 casos), com doenças oculares diversas, o estudo seria fortalecido com aumento amostral, e maior "follow-up" dos pacientes para reaplicação do instrumental de qualidade de vida. Os resultados se referem a um período de apenas três meses, considerado pequeno para casos mais graves.
Outras variáveis devem ser administradas e podem influenciar a qualidade de vida relacionada à visão(10) e a resposta da criança ao tratamento, tais como as condições neurológicas e/ou síndromes associadas, expectativas dos pais, condição socioeconômica e aderência e assiduidade ao tratamento.

A melhora constatada nos escores de impacto familiar pode se explicar pelo acolhimento da família em um serviço de atendimento de reabilitação visual da criança e assistência aos familiares. 0 momento de diagnóstico pode ser emocionalmente desestruturante para as mães, evidenciando a importância da estimulação adequada à criança com baixa visão(4) . O processo de reabilitação visual auxilia na diminuição de fatores depressivos e ansiógenos desencadeados pela situação de crise inicial.

A estimulação visual requer colaboração e bom vínculo entre pais e terapeutas, com a finalidade única de favorecer o desenvolvimento neuropsicomotor e o uso funcional da visão pela criança $a^{(18)}$. Aos poucos, em contato com os profissionais de reabilitação, a família começa a se adaptar e buscar ajuda, pois percebe que as crianças apresentam necessidades visuais específicas ${ }^{(19)}$ e precisam de tratamentos o mais rápido possível. Com o vínculo mais fortalecido e uma visão mais realista das possibilidades e potencialidades da criança, os pais começam a aceitá-la.

As pesquisas relacionadas ao tratamento de estimulação visual precoce e seus efeitos são escassas, o que evidencia a importância deste estudo na área de reabilitação, com o intuito de fornecer dados sobre evolução do tratamento e a eficácia da conduta adotada. É indispensável que os novos estudos demonstrem a importância da avaliação e intervenção precoces, para prevenir os possíveis atrasos ocasionados pela baixa visão(20).

\section{CONCLUSÃO}

A qualidade de vida relacionada à visão de crianças com deficiência visual atendidas no Ambulatório de Estimulação Visual Precoce da Unifesp, comparando-se os dois momentos, antes e após intervenções reabilitacionais de equipe multiprofissional, apresentou melhora com diferença estatisticamente significante na saúde geral da visão.

As intervenções de equipe multidisciplinar em baixa visão promoveram melhora na qualidade de vida das crianças e suas famílias.

Este estudo achou recomendável a realização de novas pesquisas, com maior número amostral e maior tempo de seguimento dos pacientes para avaliar a importância da avaliação precoce da visão em crianças e suas repercussões na qualidade de vida.

$O$ atendimento multiprofissional por equipe especializada e a reavaliação destes casos utilizando os mesmos instrumentos é essencial para se quantificar a eficácia e importância da intervenção.

Quanto mais precoce o diagnóstico e o tratamento em reabilitação, maiores as possibilidade de prevenção dos atrasos ocasionados pela deficiência visual. A adesão e assiduidade ao tratamento são 
influenciados pela colaboração da família e do bom vínculo com a equipe terapêutica.

O instrumento mostrou-se útil para o objetivo do estudo, apresentando correlações significativas em quase todas as subescalas, entre os dois momentos.

\section{REFERÊNCIAS}

1. Cobo AD, Rodriguez MG, Bueno ST. Personalidade e auto-imagem do cego. In: Martin MB, Bueno ST. Deficiência visual: aspectos psicoevolutivos e educativos. Trad. Magali de Lourdes Pedro. São Paulo: Santos; 2003.

2. Graziano RM, Leone CR. Problemas oftalmológicos mais frequentes e desenvolvimento visual do pré-termo extremo. J Pediatr. 2005:81(1 Supl):S95-100.

3. Malta J, Endriss D, Rached S, Moura T, Ventura L. Desempenho funcional de crianças com deficiência visual, atendidas no Departamento de Estimulação Visual da Fundação Altino Ventura. Arq Bras Oftalmol. 2006:69(4):571-4.

4. Figueiredo MO, Paiva e Silva RB, Nobre MI. Diagnóstico de baixa visão em crianças: sentimentos e compreensão de mães. Arq Bras Oftalmol. 2009;72(6):766-70.

5. Núñez B. La familia con un hijo con discapacidad: sus conflictos vinculares. Arch Argent Pediatr. 2003;101(2):133-42.

6. Osaki MH, Belfort Junior R. Qualidade de vida e custos diretos em pacientes com blefaroespasmo essencial e espasmo hemifacial, tratados com toxina botulínica-A. Arq Bras Oftalmol. 2004;67(1):43-9.

7. Scott IU, Smiddy WE, Schiffman J, Feuer WJ, Pappas CJ. Quality of life of low-vision patients and the impact of low vision services. Am J Ophthalmol. 1999;128(1):54-62.

8. Bittencourt ZZ, Hoehne EL. Qualidade de vida de deficientes visuais. Medicina (Ribeirão Preto). 2006:39(2):260-4

9. Lopes MCB, Salomão SR, Berezovsky A, Tartarella MB. Avaliação da qualidade de vida relacionada à visão em crianças com catarata congênita bilateral. Arq Bras Oftalmol. 2009:72(4):467-80

10. Chadha RK, Subramanian A. The effect of visual impairment on quality of life of children aged 3-16 years. Br J Ophthalmol. 2011;95:642-5.

11. Mello PR, Roma AC, Moraes Júnior HV. Análise da qualidade de vida de portadores de uveítes de causas infecciosas e não infecciosas pelo questionário NEI-VFQ-25. Arq Bras Oftalmol. 2008,71(6):847-54.

12. Moreira LB, Alchieri JC, Belfort Junior R, Moreira H. Aspectos psicossociais do paciente com ceratocone. Arq Bras Oftalmol. 2007;70(2):317-22.

13. Lucas MB, Leal DB, Tavares SS, Barros EA, Aranha ST. Condutas reabilitacionais em pacientes com baixa visão. Arq Bras Oftalmol. 2003;66(1):77-82.

14. Felius J, Starger DR Sr, Berry PM, Fawcett SL, Starger DR Jr, Salomão, S, et al. Development of an instrument to assess vision-related quality of life in young children. Am J Ophtalmol. 2004;138(3):362-72.

15. Souza TA, Souza VE, Lopes MCB, Kitadai SP. Descrição do desenvolvimento neuropsicomotor e visual de crianças com deficiência visual. Arq Bras Oftalmol. 2010;73(6):526-30.

16. Navarro AS, Fontes SV, Fukujima MM. Estratégias de intervenção para habilitação de crianças deficientes visuais em Instituições especializadas: estudo comparativo. Rev Neurociências. 1999;7(1):13-21.

17. Mérula RV, Fernandes LC. Catarata infantil: importância do diagnóstico e tratamento precoces. Arq Bras Oftalmol. 2005;68(3):299-305.

18. Gagliardo HG, Nobre MI. Intervenção precoce na criança com baixa visão. Rev Neurociências. 2001;9(1):16-9.

19. Figueiredo MO, Paiva e Silva RB, Nobre MI. Mães de crianças com baixa visão: compreensão sobre o processo de estimulação visual. Rev Psicopedagogia. 2011;28(86): $156-66$.

20. Pinheiro RC, Pizzani L, Martinez CMS, Hayashi MCPI. Produção científica sobre avaliação da visão em crianças: um estudo bibliométrico na base de dados LILACS. Rev Educ Esp. 2012;25(42):143-66.

\title{
$38 .{ }_{20 n g r e s s o d a}$ \\ $38^{\circ}$ Congresso da \\ Associação Paranaense de Oftalmologia
}

\author{
14 a 16 de março de 2013 \\ Expo Unimed - Asa 2 \\ Curitiba (PR)
}

Informações:

Tels.: (41) 3232-4031 ou 3039-8003

Site: www.apo-pr.com.br 